\title{
Cardiac involvement in polymyositis: Report of case and review of literature
}

\author{
DENNIS W. RHOADES, D.O. \\ Reynoldsville, Pennsylvania \\ RICHARD A. PASCUCCI, D.O. \\ East Lansing, Michigan
}

Polymyositis and dermatomyositis may be described as inflammatory disorders of skeletal musculature that result in symmetric weakness and/or atrophy of the proximal hip and shoulder girdles. ${ }^{1,2}$ These diseases are fairly well defined by criteria ${ }^{3}$ adapted from Bohan and Peter (1975), which include proximal muscle weakness, serum muscle enzyme elevation (particularly creatine kinase $C K$ ), typical electromyographic changes, characteristic muscle biopsy pathology, and classic dermatologic findings. Many extramuscular manifestations, including articular symptoms, ${ }^{4}$ pulmonary interstitial disease ${ }^{5}$ decreased gastrointestinal motility, ${ }^{6}$ and Raynaud's phenomenon, ${ }^{7}$ have been described.

Dermatomyositis has also been associated with an underlying malignancy in as many as 10-30 percent of adult patients, especially patients over
40 years of age. It is often mentioned that males may predominate, but recent studies have shown that females may be affected in the majority of cases. ${ }^{8-10}$

Cardiac involvement in polymyositis, although first described by Oppenheim ${ }^{11}$ as early as 1899 , was thought ${ }^{12,13}$ to be a rare manifestation of this disorder until recent studies ${ }^{14-17}$ emphasized the frequency of atrial and ventricular dysrhythmias, ${ }^{15}$ cardiac conduction defects, ${ }^{18}$ hyperkinetic heart disease, ${ }^{19}$ congestive heart failure, ${ }^{16}$ mitral valve prolapse, ${ }^{15}$ and pericarditis ${ }^{20}$ (Table 1). Postmortem studies $^{16,21}$ have noted the prevalence of inflammatory and fibrotic changes in the myocardium and conduction system, as well as the rare finding of coronary artery vasculitis. ${ }^{21}$ These histopathologic changes seem to correlate well with the various clinical presentations ${ }^{16,17,21}$ and are analogous to the inflammatory changes noted in proximal skeletal muscles.

The following report describes an adult with primary idiopathic polymyositis. Not only did electrocardiographic changes related to his underlying muscular disease develop, but he was incidentally noted to have hypertrophic cardiomyopathy.

\section{Report of case}

A 53-year-old black man presented with a 4-week history of symmetric polyarthritis and mild myositis, affecting the shoulders, wrists, hands, elbows, and ankles, as well as a mild muscle pain. He complained of morning stiffness lasting approximately 1 hour, and positive physical findings included a grade $2-3 / 6$ aortic systolic murmur, bibasilar rales in the lungs, and mild synovitis with thickening of wrists, MCP and PIP joints bilaterally. Small knee effusions were present as well. No skin rash, alopecia, or mouth lesions were noted.

The creatine kinase (CK) value was 1,725 I.U. (normal, 80-180), the serum glutamic-oxaloacetic transaminase level was 91 I.U. (normal, 10-40), and the serum glutamicpyruvic transaminase value was 105 I.U. (normal, 5-45). Lactic dehydrogenase (LDH) was 440 I.U. (normal, $110-220)$ and the erythrocyte sedimentation rate (ESR) 


\begin{tabular}{|ll|}
\hline TABLE 1. CARDIAC ABNORMALITIES IN POLYMYOSITIS. \\
\hline Type of finding & Abnormality \\
\hline Electrocardiographic & Dysrhythmia \\
& Conduction defect \\
& Left atrial abnormality \\
& Nonspecific ST-T wave \\
& Old myocardial infarction \\
& Axis deviation \\
Echocardiographic & Mitral valve prolapse \\
& Hyperkinetic cardiac state \\
& Pericarditis (rare) \\
Clinical & Systolic murmur (mitral) \\
& Congestive heart failure \\
Pathologic & Myocarditis \\
& Fibrosis \\
& Coronary vasculitis (rare) \\
\hline
\end{tabular}

was $124 \mathrm{~mm}$./hour. Bibasilar pulmonary fibrosis was noted on routine chest $\mathrm{x}$-ray examination. The EKG revealed no sinus rhythm at 90 beats per minute with nonspecific ST-T wave changes.

The patient was a moderate to heavy drinker (approximately $1,500 \mathrm{ml}$. of rum per week). He was treated with Clinoril (200 mg. twice a day) for tentatively diagnosed rheumatoid arthritis with mild myositis. He was instructed to refrain from alcohol.

He returned to the hospital 8 weeks later because of progression of symptomatology, stating that he had completely abstained from alcohol. At this time, a rheumatology consultant also found symmetric polyarthritis, as well as severe proximal myopathy (Grade II/V). Laboratory data included a $\mathrm{CK}$ value of $>15,000$ I.U. and a serum aldolase level of 135 units (normal 0-6 units). A unilateral electromyogram yielded myopathic changes characteristic of polymyositis; biopsy of the right deltoid muscle showed inflammatory cellular infiltrate, patchy necrosis and fibrosis of muscle fibers, and variation of fiber size. A thyroid profile, including determinations of triiodothyronine, thyroxine, free thyroxine index, and thyroid-stimulating hormone, was normal, and Toxoplasma antibody titers (IgG and IgM) were negative. Other diagnostic studies, including complete gastrointestinal series and intravenous pyelography (to exclude occult cancer), were unremarkable.

There was no prior history of atypical chest pain, angina pectoris, hypertension, diabetes, or myocardial infarction. An electrocardiogram (EKG) that had been obtained 5 years prior to admission was normal. The present EKG again revealed nonspecific ST-T wave changes, a left atrial abnormality (P-wave mitrale), and the suggestion of an old myocardial infarction (QS complexes in precordial leads). Physical examination also revealed an aortic, systolic murmur, varying in intensity. Echocardiography disclosed systolic anterior motion of the anterior mitral valve leaflet with concentric left ventricular hypertrophy, which is in keeping with a diagnosis of hypertrophic cardiomyopathy. ${ }^{22,23}$ Therapy was initiated with $60 \mathrm{mg}$. of prednisone (drug of choice) per day in divided doses with significant improvement in symptomatology noted at 2 weeks.

\section{Discussion}

Evidence for cardiac involvement in polymyositis has been greatly enhanced by the utilization of current, noninvasive techniques. The routine EKG has disclosed abnormalities from 33 to 70 percent of patients, ${ }^{16,24}$ with nonspecific ST-T wave changes, left atrial abnormalities, axis deviation, and varying degrees of atrioventricular block being recognized most frequently. ${ }^{16}$ Complete heart block necessitating pacemaker insertion has even been reported. ${ }^{12}$ Other significant findings have included atrial and ventricular dysrhythmias as well as EKG evidence of old myocardial infarction ${ }^{16,25}$ (in 1 case $^{25}$ despite normal coronary arteries at angiography). One investigator ${ }^{26}$ has shown that continuous 24-hour EKG monitoring (Holter) may even increase the diagnostic yield of potentially serious dysrhythmias in polymyositis, thereby recommending periodic Holter monitoring of this group of patients.

Gottdiener and coworkers ${ }^{15}$ have employed the echocardiogram and phonocardiogram in addition to the EKG in their prospective evaluation of cardiac function in polymyositis. Analysis of their findings unveiled evidence of cardiac abnormalities in 76 percent of patients $(16 / 21)$, with an overall incidence of mitral valve prolapse in 65 percent (11 of 17 studied). Further evidence for the hyperkinetic cardiac state characterized by enhanced left ventricular systolic function in the etiology of congestive heart failure also was discussed.

Askari ${ }^{26}$ prospectively evaluated 8 patients with polymyositis who had elevation of the CK-MB isoenzyme fraction to $>3$ percent of the total CK. Not only were EKG, Holter monitor, and echocardiogram recorded, but thallium-201 scintiscan and gated blood pool studies were performed. The inclusion of these noninvasive scanning techniques led to the detection of rather subtle myocardial abnormalities, which in turn led to the diagnosis of cardiac involvement in 100 percent of patients. Either patient selection (elevated CK-MB fraction) or improved diagnostic techniques may well have been responsible for this diagnostic frequency. Askari also suggested that elevation of the CK-MB band in this subgroup of patients served as an indicator of poor prognosis.

The patient in the case reported here certainly exemplifies some of the common cardiac abnormalities of polymyositis by the development of various EKG abnormalities in concert with the onset of inflammatory muscular disease. The electrical evidence of nonspecific ST-T wave changes, left atrial abnormality, and old myocardial infarction could theoretically be attributed to his hypertrophic cardiomyopathy, except that these findings were ab- 
sent prior to the development of polymyositis. One could also reason that alcoholic myopathy, which has been well described ${ }^{27-29}$ and is often difficult to distinguish from polymyositis, was the cause of his muscular disorder. The patient, however, exhibited bibasilar pulmonary fibrosis, symmetric polyarthritis, and disease progression (CK elevation from 1,700 to $>15,000$ I.U.) despite abstinence from alcohol. These features, as well as the cardiac findings, are more characteristic of a connective tissue disorder than of acute or chronic alcoholic myopathy. Also, there was no echocardiographic evidence of alcoholic cardiomyopathy.

The histologic features of hypertrophic cardiomyopath ${ }^{22}$ differ significantly from those of polymyositis, so a cause-effect relationship is difficult to postulate as the sole explanation for the cardiac picture in this case. Recent evidence, ${ }^{30,31}$ however, has also noted the association between cardiac and skeletal muscle myositis, supporting the theory that cardiomyopathy in some cases may be a partial clinical feature of a generalized myopathy. Nonetheless, this would appear to be one of the first reported associations of hypertrophic cardiomyopathy with polymyositis.

\section{Comment}

Cardiac involvement in polymyositis is remarkably more common than previously presumed. This extramuscular manifestation is now recognized in the majority of patients. The use of noninvasive cardiac studies, such as EKG, chest x-ray, CK-isoenzymes, Holter monitor, and echocardiogram, may better define polymyositis cardiac involvement and, therefore, guide its therapy. The specific management of the patient with an elevated CKMB band may be enhanced further by use of noninvasive scanning (thallium-201 and gated blood-pool studies).

Treatment of a cardiac manifestation of polymyositis usually consists of aggressive control of the underlying disease process by utilization of corticosteroids and/or immunosuppressive agents, when indicated. Further therapy is individualized and dependent upon the specific clinical settingfor example, antiarrhythmics for dysrhythmias, pacemaker for significant heart block, and diuretics for congestive heart failure. Through an increased awareness of cardiac involvement and the appropriate use of diagnostic and therapeutic modalities as described, it is anticipated that decreased morbidity and mortality from polymyositic heart disease might result.

1. Bohan, A., and Peter, J.B.: Polymyositis and dermatomyositis (First of two parts). N Engl J Med 292:344-7, 13 Feb 75
2. Bohan, A., and Peter, J.B.: Polymyositis and dermatomyositis (Second of two parts). N Engl J Med 292:403-7, 20 Feb 75

3. Hudgson, P., and Peter, J.B.: Inflammatory disorders of muscle. Classification. Clin Rheum Dis 10:3-8, Apr 84

4. Spiera, H.: Polymyositis and other diseases of muscle. In Rheumatic diseases: Diagnosis and management, edited by W.A. Katz. J.B. Lippincott Co., Philadelphia, 1977

5. Songcharoen, J., Raju, S.F., and Pennebaker, J.B.: Interstitial lung disease in polymyositis and dermatomyositis. J Rheumatol 7:353-60, May-Jun 80

6. De Merieux, P., et al.: Esophageal abnormalities and dysphagia in polymyositis and dermatomyositis. Clinical, radiographic, and pathologic features. Arthritis Rheum 26:961-8, Aug 83

7. Pearson, C.M.: Polymyositis and dermatomyositis. In Arthritis and allied conditions: A textbook of rheumatology, edited by D.J. McCarty. Ed. 9. Lea \& Febiger, Philadelphia, 1979

8. Barnes, B.E.: Dermatomyositis and malignancy. A review of the literature. Ann Intern Med 84:68-76, Jan 76

9. Callen, J.P., et al.: The relationship of dermatomyositis and polymyositis to internal malignancy. Arch Dermatol 116:295-8, Mar 80

10. Manchul, L.A., et al.: The frequency of malignant myeloma in patients with polymyositis-dermatomyositis. A controlled study. Arch Intern Med 145:35-9, Oct 85

11. Oppenheim, H.: Zur dermatomyositis. Berl Klin Wochenschr 36:805-7, 11 Sep 1899

12. Schaumburg, H.H., Neilson, S.L., and Yurchak, P.M.: Heart block in polymyositis. N Engl J Med 284:480-1, 4 Mar 71

13. Lynch, P.G.: Cardiac involvement in chronic polymyositis. Br Heart J 33:416-9, May 71

14. Askari, A.D., and Huettner, T.L.: Cardiac abnormalities in polymyositis/dermatomyositis. Semin Arth Rheum 12:208-19, Nov 82

15. Gottdiener, J.S., et al.: Cardiac manifestations in polymyositis. Am J Cardiol 41:1141-9, Jun 78

16. Denbow, C.E., et al.: Cardiac involvement in polymyositis. A clinicopathologic study of 20 autopsied patients. Arthritis Rheum 22:1088-92, Oct 79

17. Oka, M., and Raasakka, T.: Cardiac involvement in polymyositis. Scand J Rheumatol 7:203-8, 1978

18. Kehoe, R.F., et al.: Cardiac conduction defects in polymyositis. Electrophysiologic studies in four patients. Ann Intern Med 94:41-3, Jan 81 19. Babka, J.C., and Pepine, C.J.: Hyperkinetic cardiovascular state in polymyositis. Chest $64: 243-6$, Aug 73

20. Bradley, W.G.: Inflammatory diseases of muscle. In Textbook of rheumatology, edited by W.N. Kelley, et al. Ed. 2. W.B.S Saunders, Philadelphia, 1985

21. Haupt, H.M., and Hutchins, G.M.: The heart and cardiac conduction system in polymyositis-dermatomyositis. A clinicopathologic study of 16 autopsied patients. Am J Cardiol 50:998-1006, Nov 82

22. Silver, M.M., and Silver, M.D.: Cardiomyopathies. In Cardiovascular pathology, edited by M.D. Silver. Churchill Livingstone, New York, 1983, vol. 1

23. D'Cruz, I.A.: Hypertrophic cardiomyopathy. In Echocardiographic diagnosis: Differential diagnosis of M-mode and two-dimensional echographic appearances, edited by I.A. D'Cruz. MacMillan Publishing Co., New York, 1983

24. Stern, R., et al:: ECG abnormalities in polymyositis. Arch Intern Med 144:2185-9, Nov 84

25. Cohn, H., and Lynfield, Y.L.: Myocardial infarction in dermatomyositis. Cutis 23:672-5, May 79

26. Askari, A.D.: Inflammatory disorders of muscle. Cardiac abnormalities. Clin Rheum Dis 10:131-49, Apr 84

27. Ekbom, K., et al.: Muscular affections in chronic alcoholism. Arch Neurol 10:449-58, May 74

28. Rubenstein, A.E., and Wainapel, S.F.: Acute hypokalemic myopathy in alcoholism. A clinical entity. Arch Neurol 34:553-5, Sep 77

29. Walton, J.N.: Inflammatory myopathies. In Disorders of voluntary muscle, edited by J.N. Walton. Ed. 4. Churchill Livingstone, New York, 1981

30. Rechavia, E., et al.: Polymyositic heart disease. Chest 88:309-11, Aug 85

31. Dunnigan, A., et al.: Cardiac and skeletal myopathy associated with cardiac dysrhythmias. Am J Cardiol 53:734-7, 1 Mar 84

Accepted for publication in February, 1986. Updating, as necessary, has been done by the authors. 
At the time this paper was written, Dr. Rhoades was a resident in general practice at Suburban General Hospital, Norristown, Pennsylvania. He is now in private practice in Reynoldsville, Pennsylvania. Dr. Pascucci is an associate professor in the Department of Internal Medicine at Michigan State University-
College of Osteopathic Medicine, East Lansing, Michigan.

Dr. Pascucci, B311 West Fee Hall, Department of Internal Medicine, Michigan State University-College of Osteopathic Medicine, East Lansing, Michigan, 48824. 